\title{
Coherent Synchrotron Radiation: Theory and Experiments
}

\author{
Courtlandt L. Bohn \\ Northern Illinois University, DeKalb, IL 60115, and Fermilab, Batavia, IL 60510
}

\begin{abstract}
Our understanding of the generation of coherent synchrotron radiation in magnetic bending systems and its impact on beam dynamics has grown considerably over the past few years. The search for understanding has brought a number of surprises, all related to the complexity of the fully self-consistent problem. Herein I survey the associated phenomenology, theory, and experiments while emphasizing important subtleties that have recently been uncovered. I conclude by speculating on courses of future investigations that may prove fruitful.
\end{abstract}

\section{INTRODUCTION}

The subject of coherent synchrotron radiation (CSR) in connection with chargedparticle beams is "classical," with work dating at least as far back as 1945 [1]. And studies of the phenomenon have proceeded outside the field of accelerator physics. One interesting example is a 1971 paper published in the Astrophysical Journal simply entitled "Coherent Synchrotron Radiation" [2]. The authors consider a ring of charged particles orbiting a pulsar and find that the ring can be unstable to clumping. Seemingly as an afterthought, they comment on the applicability of the instability to accelerators, saying, "it seems unlikely that the instability will be important for highenergy particle machines." While the statement was made in connection with circular machines in which the beam pipe is small enough to shield CSR, it is nonetheless "interesting" when juxtaposed against the recently discovered microbunching instability that will be described herein. The lesson to be learned is that there is much benefit to be gained in cross-fertilizing among diverse fields.

That said, CSR is of practical concern in that it can severely degrade beam quality. For example, it can change the distribution of particle energies inside an achromatic bending system, thereby ruining the achromaticity, introducing a correlated head-totail warp in a bunch, and increasing its projected transverse emittance. Its impact can be even more dramatic, resulting for example in the aforementioned microbunching. The associated fine structure can potentially mix away over relatively short time scales and degrade the slice emittance. Because bunch compressors normally comprise a series of dipole bending magnets, the phenomenon is a priori a concern for machines requiring a tight beam phase space, such as beam-driven short-wavelength light sources and linear colliders. Moreover, recent efforts to develop high-average-current recirculating accelerators, in which the beam pipe must be relatively large to avoid beam loss and vacuum-chamber shielding is correspondingly small, likewise bring 
concern about CSR-induced emittance growth. I highlighted the latter concern at the 1995 Micro Bunches Workshop [3]. Herein I highlight the contemporary understanding of beam self-interaction via CSR developed over the intervening years.

\section{ELEMENTARY PHENOMENOLOGY}

When accelerated, a system of $N$ charged particles radiates coherently at wavelengths comparable to or longer than the system size. The radiated power scales as $N^{2}$, in contrast to incoherent synchrotron radiation for which the power scales as $N$. Modern high-brightness electron beams comprise bunches with $N \sim 10^{10}$, and the coherent radiation is correspondingly strong. The bunch length can be sub-mm, corresponding to far-infrared (FIR) coherent radiation. In this regime the radiation spectrum is independent of the beam energy. At wavelength $\lambda$, the opening angle in a bend of radius $R$ is likewise energy-independent, being roughly equal to $(0.5 \lambda / R)^{1 / 3}$ [4], a value much wider than the $1 / \gamma$-dependent opening angle we are conditioned to think about in connection with short-wavelength incoherent synchrotron radiation. For example, with $\lambda=1 \mathrm{~mm}$ and $R=20 \mathrm{~cm}$, the opening angle is $\sim 8^{\circ}$.

Consider an electron bunch that has moved in free space from a straight path onto an arc, as depicted in Fig. 1. Whereas the radiation moves along a straight path at speed $c$, the bunch moves on a curved path at speed less than $c$. Accordingly, due to causality, a given electron cannot interact with itself. However, photons emitted from electrons in the tail at an earlier time $t^{\prime}$ can catch up to electrons in the head at the present time $t$, the net effect being a deceleration of the tail and acceleration of the head, i.e., a correlated energy spread. After the bunch passes through an "overtaking length" $L=2\left(3 \ell_{b} R^{2}\right)^{1 / 3}$, in which $\ell_{b}$ is a measure of the full bunch length and $R$ denotes the bend radius, it experiences a steady-state self-interaction.

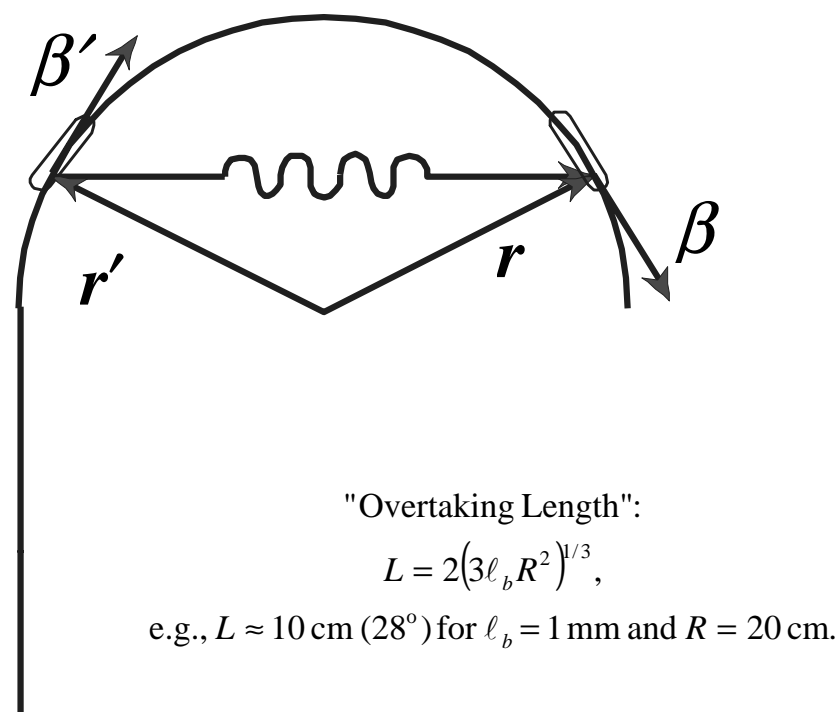

FIGURE 1. Bunch tail-to-head self-interaction via coherent synchrotron radiation. Primed variables correspond to retarded times, and $\beta=\mathbf{v} / \mathrm{c}$. 
This process has practical consequences. One example is a bending system that, per linear optics, is achromatic. Energy spread induced during transit through the bending system destroys its achromaticity and results in a warped bunch.

The presence of metallic vacuum-chamber walls complicates the interaction. For example, as shown in Fig. 2, parallel conducting plates reflect the radiation in a manner that can be represented by an infinite array of image charges. Since the path lengths of reflected photons can be long, a given electron can now interact with itself, and the head can interact with the tail. Proximity of the vacuum-chamber walls to the beam will shield the CSR. The criterion for shielding is that the factor $\eta \equiv$ $\left(2 \pi^{3} / 3\right)^{1 / 2}(R / h)^{3 / 2}\left(\sigma_{z} / R\right)$ exceed unity, wherein $h$ denotes the minimum dimension of the chamber and $\sigma_{z}$ denotes the root-mean-square (rms) bunch length.

A semianalytic calculation of the rate at which a gaussian bunch loses kinetic energy as it moves along the trajectory of Fig. 1 on the mid-plane between the two infinite plates of Fig. 2 illustrates the transient interaction with shielding. The result, extracted from [5], appears in Fig. 3, which is a plot of the lost power, normalized to that in free space, versus the angular location of the bunch centroid from the beginning of the arc. Parameters for the plot are: $R=1 \mathrm{~m}, \sigma_{z}=1 \mathrm{~mm}$, and $40 \mathrm{MeV}$ beam energy. The corresponding shielding parameter is $\eta=0.0045 / h^{3 / 2}$, so that $h=2 \mathrm{~cm}$ yields $\eta=$ 1.6 , in keeping with the strong shielding shown for that case in the Figure. The corresponding overtaking angle is $L / R=2\left\{6[2 \ln (2)]^{1 / 2} \sigma_{z} / R\right\}^{1 / 3}=0.384 \mathrm{rad}=22^{\circ}$, upon which the radiated power assumes a constant value, also in keeping with the Figure. In the transient regime, however, the power loss can exceed that of the steady state, so when dealing with short magnets, one must not presume a priori that the steady-state interaction is a good foundation for modeling the dynamics.

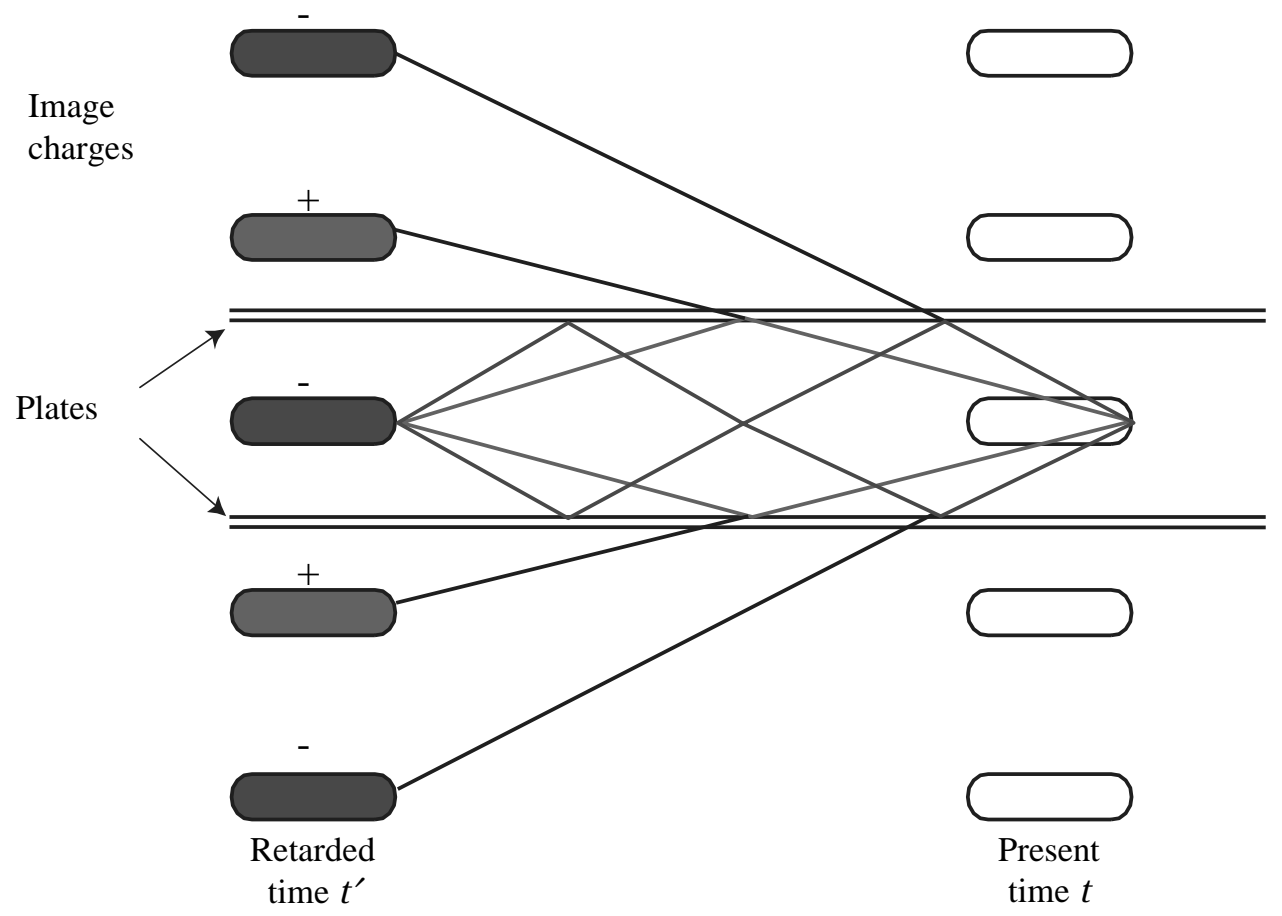

FIGURE 2. Bunch self-interaction in a vacuum chamber. 


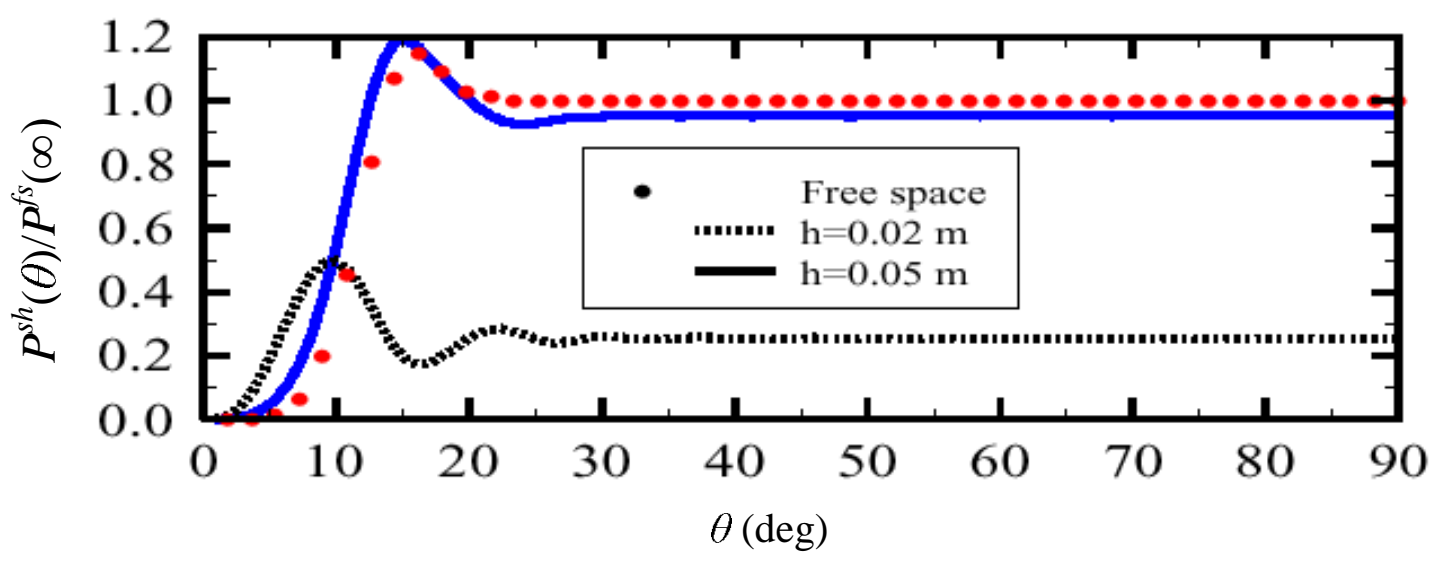

FIGURE 3. Rate of kinetic-energy loss with shielding, normalized to steady state $(t \rightarrow \infty)$, free space $(h \rightarrow \infty)$, from a bunch entering a circular arc from a straight path, versus angle into the arc (from [5]).

\section{EQUATIONS OF MOTION AND CANCELLATIONS}

The equations of particle motion in the presence of CSR follow from a concise Hamilton-Lagrange formulation. The Hamiltonian is:

$$
\text { 政 }=c \sqrt{\left(\boldsymbol{P}-\frac{e}{c} \boldsymbol{A}\right)^{2}+m^{2} c^{2}}+e \phi=c \boldsymbol{P} \cdot \boldsymbol{\beta}-\mathfrak{I},
$$

in which $m$ and $e$ are the electron's rest mass and charge, respectively, $\boldsymbol{P}=\boldsymbol{p}+(e / c) \boldsymbol{A}$ is the conjugate momentum, $\mathbb{Z}$ is the Lagrangian, and $(\phi, A)$ is the 4-potential given by

$$
\left[\begin{array}{l}
\varphi(\boldsymbol{r}, t) \\
\boldsymbol{A}(\boldsymbol{r}, t)
\end{array}\right]=e \int d \boldsymbol{r}^{\prime} d t^{\prime} \frac{\delta\left(t-t^{\prime}-\frac{1}{c}\left|\boldsymbol{r}-\boldsymbol{r}^{\prime}\right|\right)}{\left|\boldsymbol{r}-\boldsymbol{r}^{\prime}\right|}\left[\begin{array}{c}
1 \\
\boldsymbol{\beta}^{\prime}
\end{array}\right] n\left(\boldsymbol{r}^{\prime}, t^{\prime}\right) .
$$

This formalism is deceptively simple. Calculating self-consistently the present-time 4potential requires integrating over the history of the motion of all of the particles, a computationally intensive task that requires sophisticated database management. The natural question to ask is: Can any simplifications be legitimately made? An answer appears in what follows. To do a strictly analytic treatment, however, one must abandon self-consistency and specify the charge distribution for all retarded times $t^{\prime}$. The most common approach is to stipulate that the bunch comprises a rigid-line charge with some fixed longitudinal density distribution. This was the approach used, for example, to develop Fig. 3.

The equations of longitudinal motion are:

Change in single-particle total energy: $\quad \frac{d \text { 通 }}{d t}=e \frac{d V}{d t} ; \quad V=\boldsymbol{\phi}-\boldsymbol{\beta} \cdot \boldsymbol{A}$.

Change in single-particle kinetic energy: $\quad m c^{2} \frac{d \gamma}{d t}=-e \frac{d \phi}{d t}+e \frac{\partial V}{\partial t}$.

\footnotetext{
${ }^{*}$ In actuality, the bunch was constrained to the trajectory of Fig. 1, having finite length and zero width. At entry to the bend the bunch "curves" and $\mathrm{d} \varphi / \mathrm{d} t$ is nonzero during this transition.
} 
The rate of work done by the bunch against itself:

$$
P(t)=-m c^{2} \int d \boldsymbol{r} \frac{d \gamma(\boldsymbol{r}, t)}{d t} n(\boldsymbol{r}, t),
$$

which is the governing equation underlying Fig. 3.

The transverse equation of motion is written in terms of cylindrical coordinates $(r, \theta)$ referenced to the design trajectory [6]. The corresponding Lagrangian is

$$
\mathcal{I}=-m c^{2} \sqrt{1-\frac{r^{2} \dot{\theta}^{2}+\dot{r}^{2}}{c^{2}}}-e\left(\phi-\frac{\dot{r}}{c} A_{r}-\frac{r \dot{\theta}}{c} A_{\theta}\right),
$$

from which the Euler-Lagrange equation of motion follows:

$$
\begin{gathered}
\frac{d}{d t}\left(\gamma m \frac{d r}{d t}\right)-\gamma_{d e s i g n} m c^{2}\left(\frac{\beta_{\theta}^{2}}{r}-\frac{\beta_{\theta} \beta_{d e s i g n}}{R}\right)=\beta_{\theta}^{2} \frac{\left(\gamma_{\text {initial }} m c^{2}+e \phi_{\text {initial }}\right)-\gamma_{\text {design }} m c^{2}}{r} \\
+e \frac{\beta_{\theta}^{2}}{r} \int_{t_{0}}^{t} d t^{\prime} \frac{\partial(\phi-\boldsymbol{\beta} \cdot \boldsymbol{A})}{\partial t^{\prime}}-e \frac{\partial(\phi-\boldsymbol{\beta} \cdot \boldsymbol{A})}{\partial r}-\frac{e}{c} \frac{d A_{r}}{d t}-e \beta_{\theta} \frac{\beta_{\theta} \phi-A_{\theta}}{r} .
\end{gathered}
$$

On the right-hand side, the first term indicates that the initial deviation of the particle's total energy from the total energy on the design orbit contributes to the transverse motion. The second term arises from the effective force parallel to $\boldsymbol{\beta}$. Deciphering the importance of the remaining terms has been controversial.

The last term is the sum of a relic of the so-called noninertial space-charge force $F^{N S C F}$, which the scalar potential $\phi$ governs, ${ }^{*}$ and the so-called centrifugal spacecharge force $F^{C S C F}$, which the vector potential $\boldsymbol{A}$ governs. Both forces reflect a logarithmic dependence on the transverse offset. Talman [7] expresses concern that $F^{C S C F}$ would significantly influence the dynamics of a coasting, steady-state, quasidirect-current beam in a storage ring. However, Lee [8] points out that the beaminduced electric potential reduces this force by a multiplicative factor $\sigma_{x} / R$, where $\sigma_{x}$ denotes the rms transverse beam size. For bunched beams, Carlsten and Raubenheimer [9] state that the presence of $F^{N S C F}$ spoils the cancellation, though Derbenev disagrees [10].

New work by Li [6] clarifies the matter. Li shows via macroparticle simulation that the last term in Eq. (7) (denoted as " $G_{r}$ " in Fig. 4) is generally negligible in both the transient and steady-state regimes. Thus, she identifies the "effective radial force," which points in the true radial direction, to be

$$
F_{r}^{e f f}=-e \frac{\partial(\phi-\beta \cdot \boldsymbol{A})}{\partial r}-\frac{e}{c} \frac{d A_{r}}{d t},
$$

and the "total radial force" to be $F_{r}^{t o t}=F^{C S C F}+F_{r}^{e f f}$. The last term in Eq. (8) is zero in steady state but nonzero in the transient regime. Fig. 4 depicts the results of Li's simulations for a $1 \mathrm{nC}$ bunch moving at $5 \mathrm{GeV}$ in free space from a straight path onto a 10-m-radius arc. The bunch exits the arc onto a straight path upon transiting $2 \mathrm{~m}$ $\left(11.5^{\circ}\right)$ of arc. The rms dimensions of the bunch are $\sigma_{x}=0.072 \mathrm{~mm}, \sigma_{z}=0.2 \mathrm{~mm}$. Fig. 4a shows steady-state forces across the bunch's longitudinal profile. The spreads

${ }^{*} F^{N S C F} \propto-\mathrm{d} \phi / \mathrm{d} t$. The integral of $F^{N S C F}$ over time comes from the longitudinal motion, which then feeds into the transverse motion as explained in [6]. The result is - $[\phi(t)-\phi(0)] ; \phi(t)$ appears in the last term of Eq. (7) and $\phi(0)$ appears as $\phi_{\text {initial }}$ in Eq. (7). 
in $F_{r}^{\text {tot }}$ and $F^{C S C F}$ are signatures of their logarithmic dependence on the transverse coordinate. That there is no discernable spread in either $F_{r}^{e f f}$ or $G_{r}$ signifies the cancellation of the logarithmic dependence. Moreover, $G_{r}$ is seen to be negligible. Forces on a single particle in the bunch are plotted in Fig. 4b, wherein it is clear that $G_{r}$ is negligible in both the transient and steady-state regimes.

This investigation answers the question posed earlier about permissible simplifications. It suggests that setting $G_{r}=0$ is generally a good approximation. This is an important finding in that the driving force in the equations of motion then involves only far-field, not near-field, CSR interactions. In turn, the finding opens new possibilities for theoretical investigations, such as two-dimensional analyses based on Vlasov's equation, as well as the development of fast simulation codes that contain all of the essential physics. Another important observation is that $F_{r}{ }_{r}$ is not necessarily negligible, yet many analyses have been predicated on the notion (or hope) that $F_{r}^{e f f}=0$ is also a good approximation.
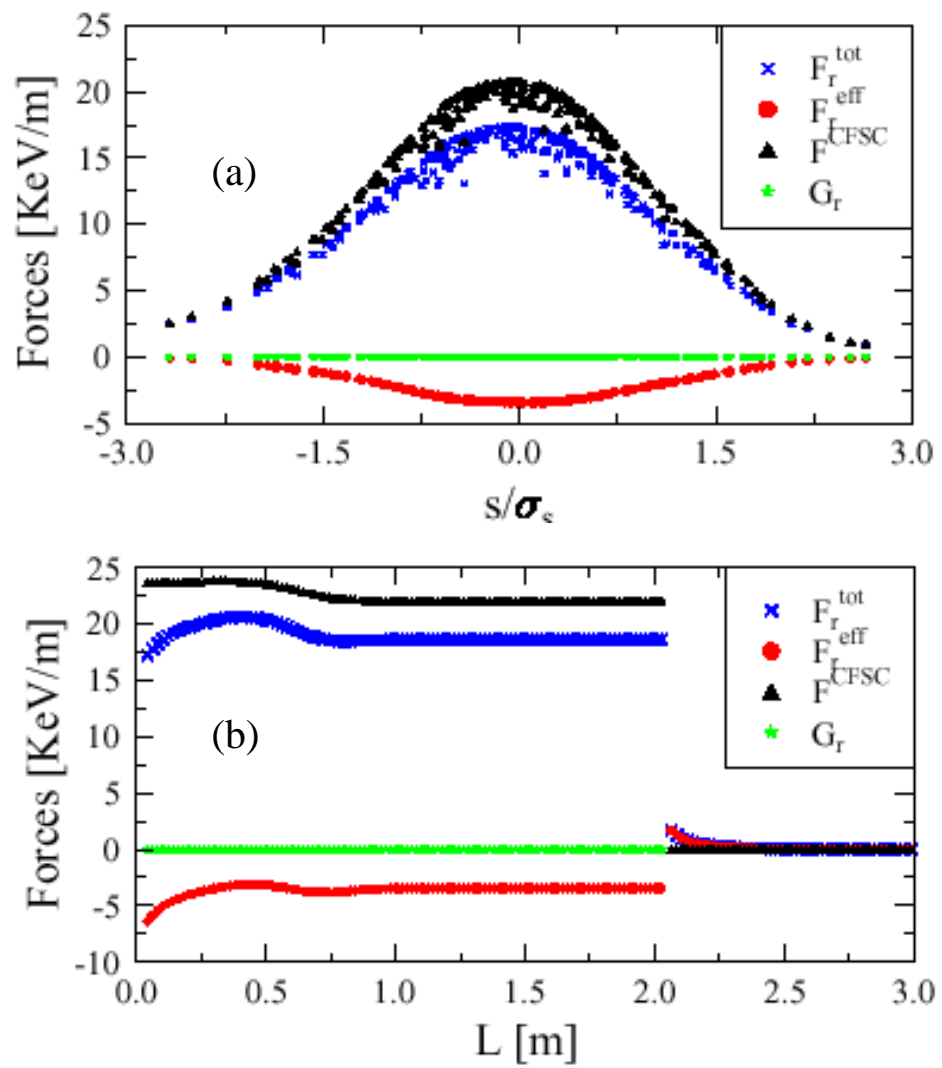

FIGURE 4. Force terms in the equation of transverse motion: (a) steady-state forces vs. longitudinal position across the bunch; (b) force terms on a single particle vs. path length. See text for details.

\section{EXPERIMENTS: BUNCH COMPRESSION, EMITTANCE GROWTH, AND MICROBUNCHING}

A bunch compressor comprises a sequence of dipole magnets, a "chicane," through which higher-energy electrons follow shorter trajectories. Prior to the 
chicane the bunch is accelerated off the radiofrequency (RF) crest so electrons in the tail gain more energy than those in the head. The tail thereby catches up to the head, resulting in a shorter bunch in keeping with a rotated longitudinal phase space.

The presence of CSR complicates matters by changing the correlated energy spread, causing the bunch to emerge with a head-to-tail "warp" and a corresponding growth in the projected transverse emittance. Even more serious, however, is the possibility of a CSR-driven instability that forms fine structure, or "microbunching," in the bunch, a phenomenon discovered only recently. For example, in shortwavelength free-electron lasers (FELs) the presence of microbunching seriously threatens to impede, or even prohibit, lasing. This matter was highlighted in a special CSR Workshop held this February in Zeuthen, Germany [11].

Measurements made in March 1999 by Piot during commissioning of the recirculation loop of Jefferson Laboratory's FEL comprise what may have been the first observation of microbunching [12]. The data appears in Fig. 5 and pertain to a $40 \mathrm{MeV}$ beam with $\sim 0.04 \mathrm{nC}$ bunch charge. Fine structure appeared in the longitudinal energy distribution in the vicinity of maximum bunch compression in the chicane labeled " $\delta 1$ ", as indicated in Fig. 5a. It was reproducible and was more enhanced at larger bunch charges. At the time of the observation, the investigating team lacked a full appreciation of the importance of the phenomenon, apart from recognizing that it implied a complicated phase space going into the chicane. Emittances versus RF phase in the linac, measured before and after the $180^{\circ}$ bend labeled "arc \#1," appear in Fig. 5b. Interestingly, the maximum emittance growth did not correspond to maximum compression (which occurred at the peak of the coherent-transition-radiation signal from the bunch-length monitor labeled "BL"). The beam waist changes through the bend, and the emittance was found to increase as the energy-phase correlation moved the longitudinal waist closer to the entrance of the arc. Subsequent analysis pointed to CSR as the most probable cause for these observations.

Compression-induced fine structure in the energy distribution has subsequently been seen at a number of facilities [11]. Using tomographic techniques, Huening has unambiguously associated the phenomenon with microstructure, or "microbunching" as it is now commonly called, in the longitudinal density profile [13]. Figure 6 depicts the results of Huening's investigation, which concerns data taken at DESY's TESLA Test Facility (TTF) with $1 \mathrm{nC}$ bunches at $135 \mathrm{MeV}$.

Especially pronounced microbunching has appeared at Brookhaven's Source Development Laboratory (SDL) [14]. Figure 7 provides an example corresponding to near-maximum compression of $0.25 \mathrm{nC}$ bunches at $75 \mathrm{MeV}$. These are direct timedomain measurements, i.e., density profiles, obtained via RF-zero phasing of the linac downstream from the compressor, after which a $70^{\circ}$ dipole magnet disperses the beam onto a scintillating screen. The investigating team suspects the microbunching is an amplification of a "seed" modulation carried by the beam on entry to the compressor. The microbunching was observed to be sensitively dependent on the phase-matching angle of the frequency-doubling crystals in the laser's optical path, and so temporal variations in the drive laser are identified as a possible source of modulation. However, there is an open question on the role, if any, of beam-generated wakefields induced by rough beam-pipe surfaces. 

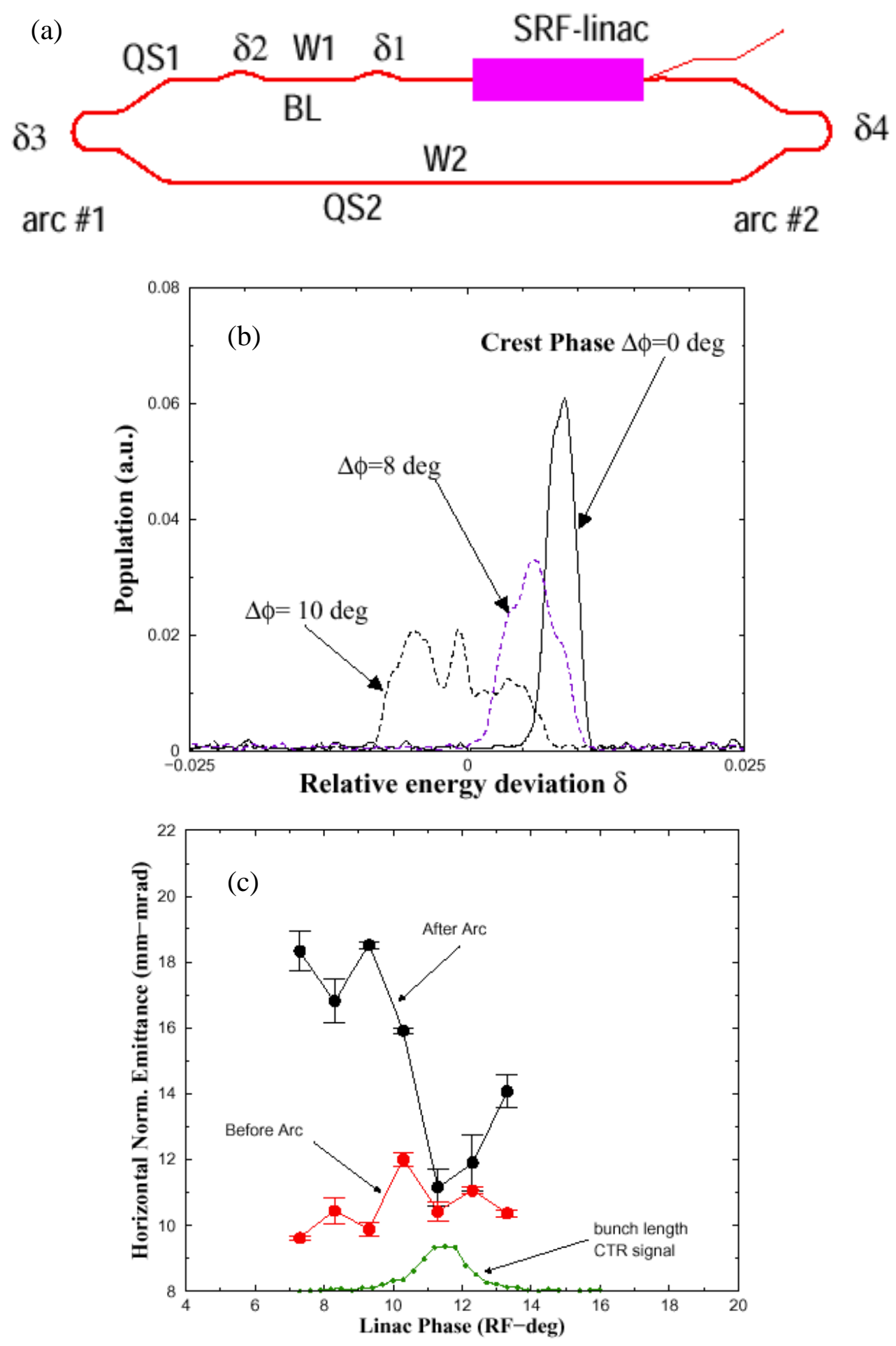

FIGURE 5. Early measurements (taken March 1999) of microbunching and emittance growth of 0.04 $\mathrm{nC}$ bunches in bends (from [12]): (a) layout of the Jefferson Lab FEL and experimental stations (see text); (b) fine structure in the energy profile appeared as the linac RF phase $\Delta \phi$ moved closer to that needed for maximum compression; (c) the transverse emittance in the bend plane of arc \#1 grew as the longitudinal waist was moved closer to the arc's entrance. 
(a)

TTF FEL 1
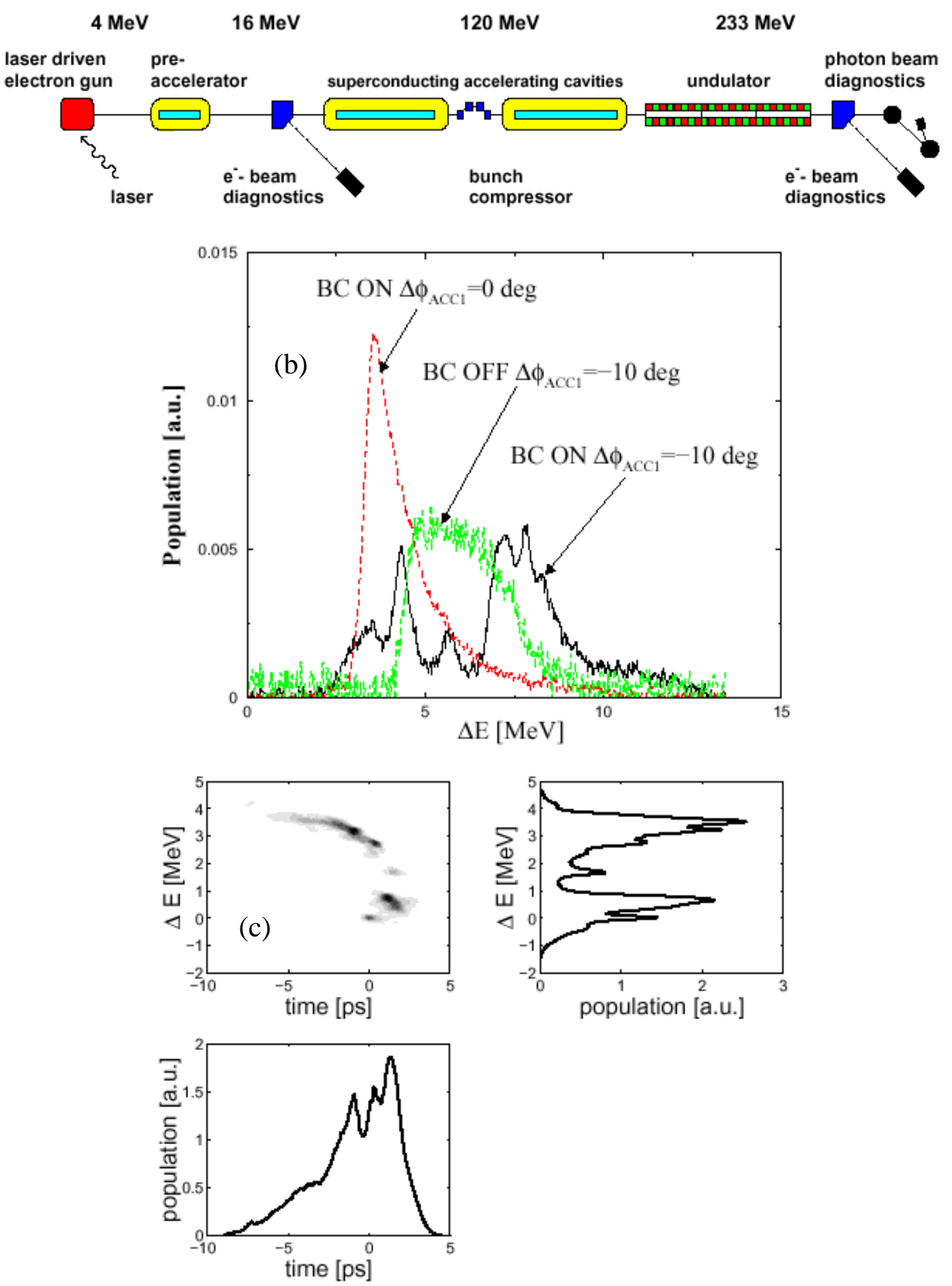

FIGURE 6. Phase-space fragmentation and microbunching of $1 \mathrm{nC}$ bunches (from [13]): (a) layout of TTF, in which bunch compression occurs at $\sim 120 \mathrm{MeV}$ (135 MeV for this data); (b) fine structure in the energy profile appeared as the linac RF phase $\Delta \phi$ moved closer to that needed for maximum compression; (c) the tomographically reconstructed phase space. 

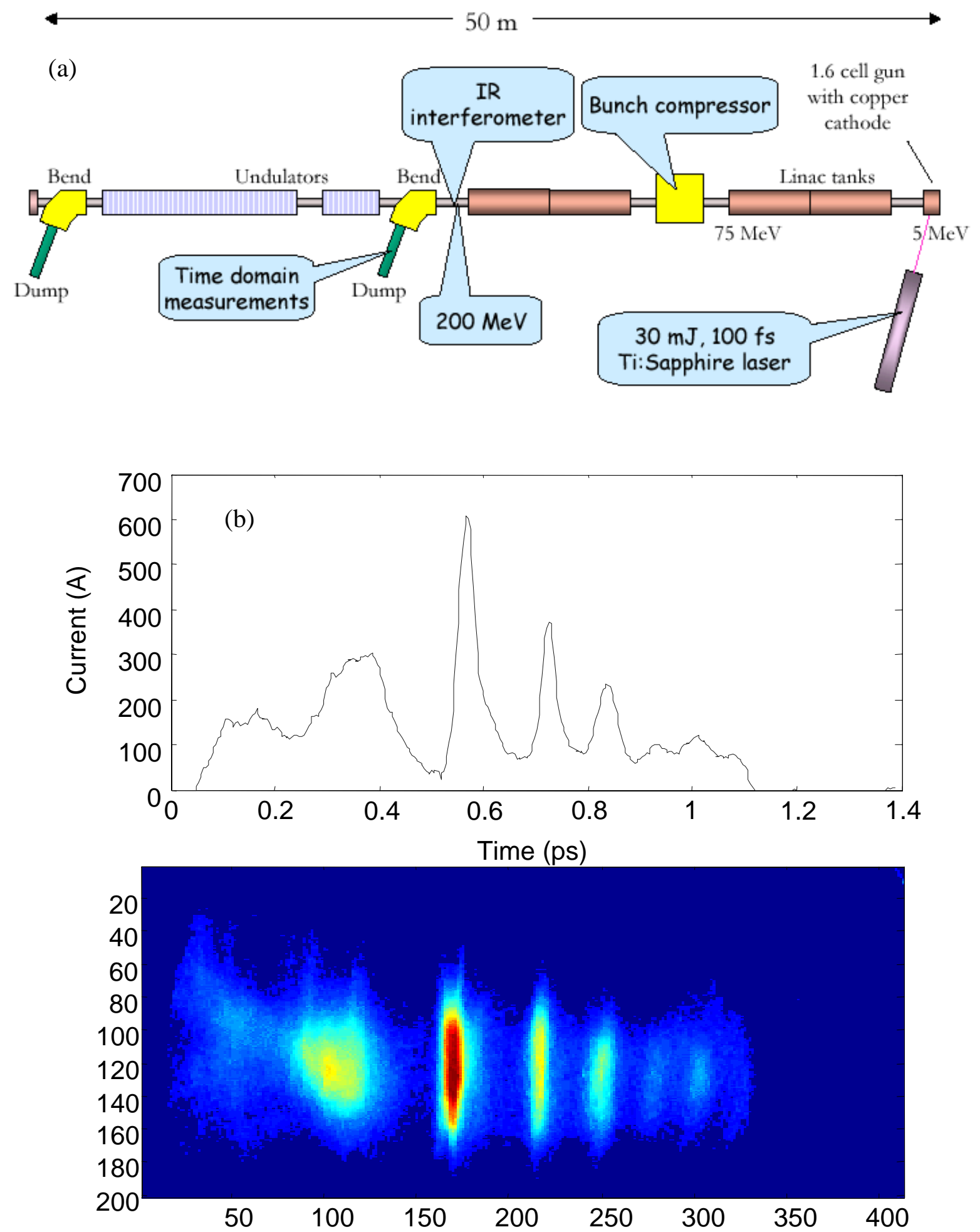

FIGURE 7. Phase-space fragmentation and microbunching of $0.25 \mathrm{nC}$ bunches (from [14]): (a) layout of SDL; bunch compression occurs at $\sim 75 \mathrm{MeV}$; (b) strong microbunching near maximum compression; temporal resolution is $\sim 50 \mathrm{fs}$; (c) corresponding projected longitudinal density. 


\section{THEORY: ENHANCED CSR AND MICROBUNCHING}

In the steady-state interaction in free space, the coherent power radiated by a rigidline gaussian bunch is (in cgs units) [15]

$$
P_{c o h}=\frac{3^{1 / 6} \Gamma^{2}(2 / 3)}{2 \pi} \frac{(N e)^{2} c}{\left(R \sigma_{z}^{2}\right)^{2 / 3}},
$$

i.e., $P_{c o h} \propto q^{2} / \sigma_{z}^{4 / 3}$, with $q$ denoting the bunch charge, indicating that the CSR power grows as the bunch length shrinks. This hints at, but does not proclaim, the sensitivity of the interaction to the detailed beam properties through the bending system.

As noted earlier, an integral part of bunch compression is off-crest acceleration. Given its finite length, a bunch acquires a curvature in its longitudinal phase space because the RF accelerating field along the bunch is nonuniform. The process of compressing the bunch can then markedly accentuate the curvature. An example is provided in Fig. 8, which depicts the outcome of simulations for zero and nonzero uncorrelated energy spread in the bunch [16]; the longitudinal phase space appears in Fig. 8a, and the corresponding density profile appears in Fig. 8b. This is a feature of the compressed beam that has aided lasing at DESY's TTF [17]. The advantage is a shorter bunch for better lasing at short wavelengths. The disadvantage is the formation of a long tail comprising a substantial fraction of the bunch charge; these "tail electrons" do not participate in the lasing process. In beams with small rms uncorrelated energy spread, the density enhancement can be pronounced, leading to a substantially stronger CSR interaction as indicated in Fig. 8c.
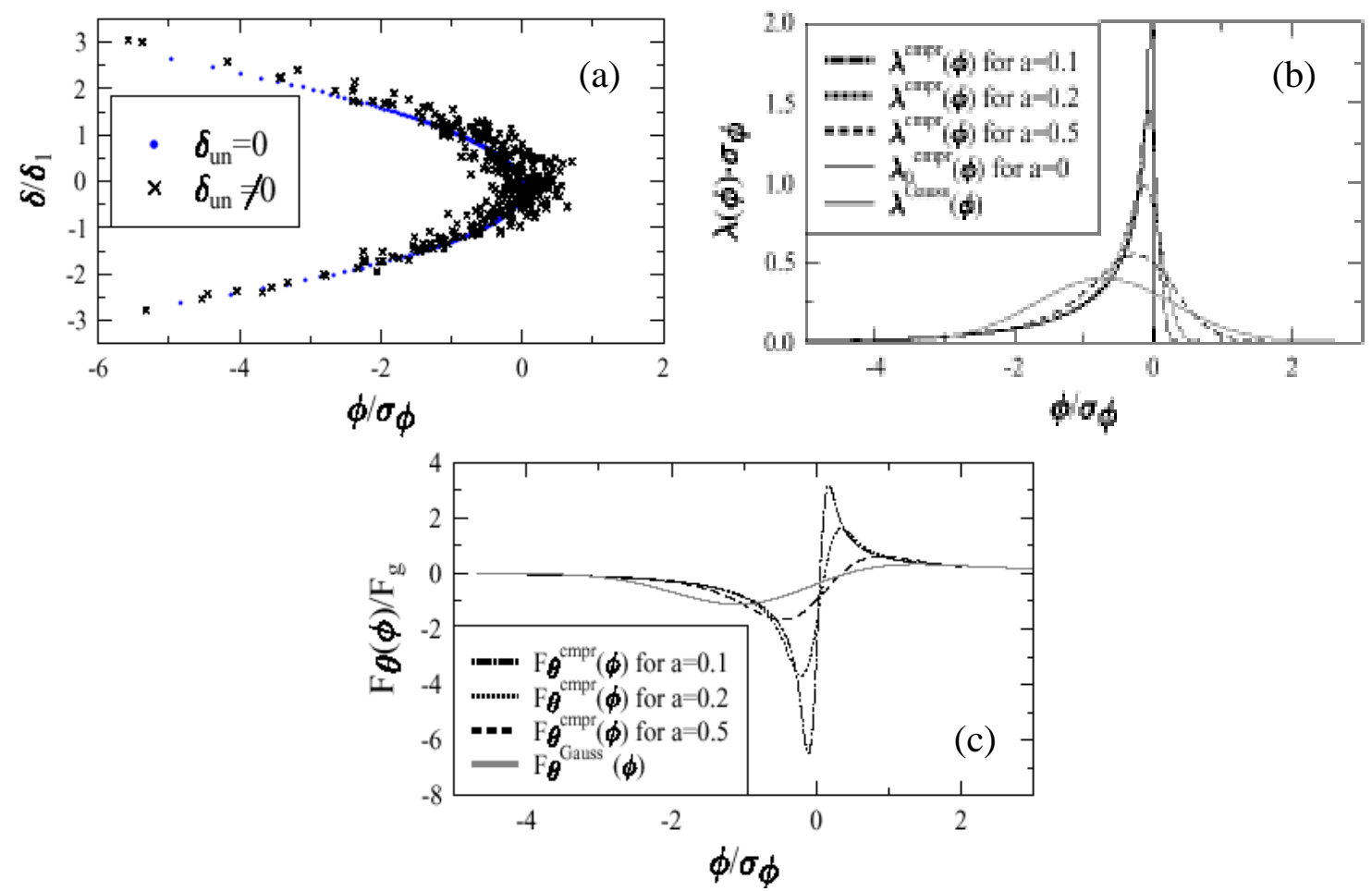

FIGURE 8. Example of longitudinal phase space distribution after compression with rf curvature (from [16]): (a) energy spread vs. position along the bunch; (b) corresponding charge distribution; (c) corresponding CSR force. 
The density structure and its influence on the CSR force exhibited in Fig. 8 point to the concern about microbunching in which there are effectively several such density peaks (see, e.g., Fig. 7). One would generally like to avoid such problems in generating high-brightness beams. One idea for removing RF-induced curvature is to use a higher-harmonic accelerating cavity prior to bunch compression [18].

Additional steps may be necessary to remove microbunching. Considerable theoretical work has recently ensued toward deciphering a viable procedure. The design of the compression chicane for the Linac Coherent Light Source (LCLS) has received special attention.

Theoretical treatments of microbunching have thus far centered on the use of a linearized Vlasov equation to extract the early evolution of a perturbation of the longitudinal density distribution. The underlying theme is that the beam's longitudinal phase space carries high-frequency "seed" modulations arising from some source, which are then amplified during bunch compression. Commonly used simplifying assumptions include: the length of the dipole magnets is small compared to the spacing between them; the CSR interaction is steady-state, not transient (the transients being more relevant at longer wavelengths); no shielding; no transverse beam size, no space charge, and no collective transverse forces. The goal is to calculate the gain, i.e., the amplification factor.

Saldin, Schneidmiller, and Yurkov treat the problem as a klystron instability and consider the case of zero compression and high gain [19]. They identify two limiting cases pertaining to whether the transverse beam size is smaller or larger than the dispersion-induced transverse size at the middle of the chicane. For large rms beam size $\sigma_{x}$, the gain in density modulation scales as $\sigma_{x}^{-8 / 3}$. This result suggests a possible cure may be to compress a beam that is large in the bend plane, such as can be produced via a "round-to-flat transformation" [20]. An experimental investigation of this possibility is under consideration at Fermilab.

Heifets, Stupakov, and Krinsky extend the treatment considerably by explicitly including bunch compression, which involves accounting for both the longitudinal and radial degrees of freedom [21]. Using the method of characteristics, they obtain a linear Volterra integral equation for the Fourier component of the density modulation. The integral equation, which they solve numerically, applies to both a microwave instability and a klystron-type amplification. Huang and Kim have very recently introduced an analytic, iterative solution of the integral equation [22].

The two teams of investigators apply their results to the second LCLS compressor for which the beam energy is $4.54 \mathrm{GeV}$, the bunch charge is $1 \mathrm{nC}$, and the bunch length begins at $195 \mu \mathrm{m}$ and ends at $23 \mu \mathrm{m}$. Both obtain the same result, pictured in Fig. 9. In the figure caption, $\sigma_{\delta}^{u n}$ and $\varepsilon$ denote the uncorrelated rms relative energy spread and the normalized rms transverse emittance, respectively. A key point evident in Fig. 9 is that Landau damping associated with $\sigma_{\delta}^{u n}$ and $\varepsilon$ tends to counter the instability. A second point is that the gain in the second compressor is modest. Huang and Kim calculate the combined effects of both LCLS bunch compressors and find the total gain can be considerably higher than shown in Fig. 9. They propose the addition of a superconducting wiggler to generate higher $\sigma_{\delta}^{u n}$ prior to the second compressor and thereby "cure" the instability via Landau damping. 




FIGURE 9. Gain, i.e., the final-to-initial amplitude ratio of perturbations of scale length $\lambda$, generated by the second bunch-compression chicane envisioned for LCLS (from [21,22]): (1) $\sigma_{\delta}^{u n}=3 \cdot 10^{-5}, \varepsilon=1$ $\mu \mathrm{m} ;$ (b) $\sigma_{\delta}^{u n}=3 \cdot 10^{-5}, \varepsilon=0$; (c) $\sigma_{\delta}^{u n}=3 \cdot 10^{-6}, \varepsilon=1 \mu \mathrm{m}$.

Work on the microbunching instability is continuing in earnest. For example, Rosenzweig brought a new preprint to the Workshop showing that, in the high-gain regime, the microbunching instability is formally similar to the FEL instability [23]. And O'Shea stated that the University of Maryland is funded to develop a mm-wave source based on amplification of a pre-seeded electron beam via the instability.

\section{SOME PERSONAL IMPRESSIONS}

The main point of the preceding discussion is that a number of interesting developments have ensued over the years subsequent to the 1995 Micro Bunches Workshop. They include: (1) acknowledgement that CSR, left unchecked, can severely degrade beam quality, a realization that has been experimentally confirmed; (2) realization that the output phase space from a magnetic bending system can depend sensitively on the input phase space, a development that has been aided by computational studies (cf. [24]); (3) improvement in the identification and understanding of cancellations of force terms and near-field CSR interactions in the equation of transverse motion; and (4) surprise in the discovery, both in simulations and experiments, of the microbunch instability. Along with new developments come new questions. I will venture some questions and ideas for future work here; the reader will no doubt be able to augment them.

On the theoretical side, the recent understanding of force cancellations, and in particular the cancellation of near-field interactions, is an important development. The notion that far-field interactions form a sufficient basis for the study of CSR effects on beams is intriguing. For one, it opens the possibility to develop simulation codes that 
are both fast and accurate, such as codes based on the Vlasov equation, thereby providing the means for efficient parametric studies.

On the experimental side, the sensitivity of the dynamics to the input phase space motivates special attention. In particular, one should take care to measure carefully the input phase space in conjunction with CSR experiments. Moreover, the formation of microstructure deserves further study. The interplay between the CSR field and conventional wakefields is an open question. One wakefield that is receiving increased attention is that due to roughness of the inside surfaces of vacuum enclosures through which the beam passes. The extent to which microbunching, CSR, and wakefields are interlinked is not well understood. In view of the uncertainty, it would be prudent to augment experimental investigations of beam dynamics and microbunching with direct measurement of the CSR spectrum from the bending system. If CSR were dominant, then its spectrum should correlate to the bunch structure. Otherwise, one must suspect other factors are significantly influencing the beam dynamics, or perhaps that the performance of the beam diagnostics is constrained. In any case, providing for ample cross-checking is wise.

The use of a higher-harmonic cavity to correct RF curvature prior to bunch compression deserves experimental confirmation. A third-harmonic cavity is an integral part of DESY's TTF-2 injector design that will be constructed to support an Xray FEL [18]. In turn, this injector should reveal the efficacy of the idea.

The microbunch instability is a concern for the production of high-brightness beams, and it needs a cure. One cure, of course, is to design bending systems that are resilient against microbunching. The ease of doing so is presently unclear. Another cure may be to manipulate the beam in a way that keeps the instability from growing. For example, one might transform the beam from round to flat, compress, then transform back from flat to round [25]. Providing a large emittance in the bend plane should mitigate the instability. A parametric study that quantifies how microbunching depends on the rms geometry of the input phase space would be useful in this context.

I close with an innovative idea advanced by T. Shintake at the 2002 CSR Workshop [11] in the spirit of making lemonade when life offers lemons. The idea is to use the microbunching instability to develop more compact FELs. In particular, using a laser and undulator (the "master oscillator"), one would purposely seed the electron beam with a periodic modulation that matches the FEL system, then accelerate the beam, compress the beam and thereby amplify the modulation, and then send the highly modulated beam through a now-much-shorter wiggler. Whether such an idea can be made to work in a controlled manner remains to be seen. Nonetheless, it is safe to project that CSR-related investigations will continue over the foreseeable future.

\section{ACKNOWLEDGEMENTS}

This paper is the product of many discussions with many colleagues over the years. I thank R. Li for providing an advance copy of her work on force cancellations, for detailed comments on this paper, and for her continuous communication over the past several years. I also thank P. Piot for numerous discussions over the years concerning subtleties of the CSR interaction. W. Graves kept me informed of his work at 
Brookhaven's SDL, and Z. Huang kindly provided an advance copy of his and K.-J. Kim's paper on microbunching theory. Finally, I thank D. Edwards for commenting on this paper. This work was supported by the U.S. Department of Education.

\section{REFERENCES}

1. Schwinger, J., "On radiation by electrons in a betatron," 1945 (unpublished).

2. Goldreich, P. and Kelley, D. A., Ap. J. 170, 463 (1971).

3. Bohn, C. L., "A Program to Research Emittance Growth in Bends," in Micro Bunches Workshop, eds. E. B. Blum, M. Dienes, and J. B. Murphy, AIP Conference Proceedings 367, New York: American Institute of Physics, 1996, pp. 391-396.

4. Jackson, J. D., Classical Electrodynamics, Chapter 14, New York: John Wiley \& Sons, 1999.

5. Li, R, Bohn, C. L., and Bisognano, J. J., "Shielded Transient Self-Interaction of a Bunch Entering a Circle from a Straight Path," in Coherent Electron-Beam X-Ray Sources: Techniques and Applications, eds. A. K. Freund, H. P. Freund, and M. R. Howells, SPIE Vol. 3154, 1997, pp. 223236.

6. Li, R, "Cancellation Effects in CSR Induced Bunch Transverse Dynamics in Bends," in Proceedings of the 2002 European Particle Accelerator Conference, Paris, France, 3-7 June 2002 (in press).

7. Talman, R., Phys. Rev. Lett. 56, 1429 (1986).

8. Lee, E. P., Part. Accel. 25, 241 (1990).

9. Carlsten, B. and Raubenheimer, T., Phys. Rev. E 51, 1453 (1995).

10. Derbenev, Ya. S. and Shiltsev, V. D., SLAC-Pub 7181, 1996 (unpublished).

11. CSR Workshop 2002, Zeuthen, Germany, 14-18 January 2002; http://www.desy.de/csr/

12. Piot, Ph., Bohn, C. L., Douglas, D. R., Krafft, G. A., Li, R. and Yunn, B. C., "Emittance and Energy Spread Studies in the Jefferson Lab Free-Electron Laser," in Proceedings of the 2000 European Particle Accelerator Conference, 2000, pp. 1546-1548.

13. Huening, M., Piot, Ph. and Schlarb, H., Nucl. Instrum. and Meth. Phys. Res. A475, 348 (2001).

14. Graves, W. S., et al., "Ultrashort Electron Bunch Length Measurements at DUVFEL," in Proceedings of the 2001 Particle Accelerator Conference, eds. P. S. Lucas and S. Webber, IEEE Cat. No. 01CH37268C, 2001, pp. 2224-2226.

15. Murphy, J. B., Krinsky, S. and Gluckstern, R. L., Part. Accel. 57, 9 (1997).

16. Li, R., "Enhancement of the CSR Self-Interaction Due to Local Longitudinal Charge Concentration," in Proceedings of the 2000 European Particle Accelerator Conference, 2000, pp. 1312-1314.

17. Limberg, T., Piot, Ph. and Schneidmiller, E. A., Nucl. Instrum. and Meth. Phys. Res. A475, 353 (2001).

18. Floettmann, K., Piot, Ph., Ferrario, M. and Grigoryan, B., "The TESLA X-FEL Injector," in Proceedings of the 2001 Particle Accelerator Conference, eds. P. S. Lucas and S. Webber, IEEE Cat. No. 01CH37268C, 2001, pp. 2236-2238.

19. Saldin, E. L., Schneidmiller, E. A. and Yurkov, M. V., "Klystron Instability of a Relativistic Electron Beam in a Bunch Compressor," in Proceedings of the 2001 Free-Electron Laser Conference, Darmstadt, Germany, 20-25 August 2001, (in press).

20. Edwards, D., Edwards, H., Holtkamp, N., Nagaitsev, S., Santucci, J., Brinkmann, R., Desler, K., Flöttmann, K., Bohnet, I., Ferrario, M., "Status of Flat Electron Beam Production," in Proceedings of the 2001 Particle Accelerator Conference, op. cit., pp. 73-75.

21. Heifets, S., Stupakov, G. and Krinsky, S., Phys. Rev. ST-AB 5, 064401 (2002).

22. Huang, Z. and Kim, K.-J., Phys. Rev. ST-AB (submitted).

23. Reiche, S. and Rosenzweig, J., "Comparison of the Coherent Radiation-Induced Microbunching Instability in an FEL and a Magnetic Chicane" (preprint dated 20 June 2002).

24. Borland, M., Phys. Rev. ST-AB 4, 070701 (2001).

25. Burov, A., Nagaitsev, S. and Derbenev, Ya., "Circular Modes, Beam Adapters and their Applications in Beam Optics,” FERMILAB-Pub-01/060-T (2001). 